\title{
Dimeticone-Based Pediculicides: A Physical Approach to Eradicate Head Lice
}

\author{
Jorg Heukelbach ${ }^{*}, 1,2$, Fabiola A. Oliveira ${ }^{2,3}$, Joachim Richter ${ }^{4}$ and Dieter Häussinger ${ }^{4}$ \\ ${ }^{I}$ Department of Community Health, School of Medicine, Federal University of Ceará, Fortaleza, Brazil \\ ${ }^{2}$ Anton Breinl Centre for Public Health and Tropical Medicine; School of Public Health. Tropical Medicine and \\ Rehabilitation Sciences, James Cook University, Townsville, Australia \\ ${ }^{3}$ Health Department of the City of Cologne, Counselling Center for Sexually Transmitted Diseases/AIDS, \\ Cologne, Germany \\ ${ }^{4}$ Clinic of Gastroenterology, Hepatology and Infectiology, University Heinrich Heine, Düsseldorf, Germany
}

\begin{abstract}
Resistance of head lice to insecticides with a neurotoxic mode of action, such as permethrin and malathion, is increasing in incidence and geographic extent. As a consequence, physically acting pediculicidal products have emerged. Dimeticones are synthetic silicone oils of low surface tension and can therefore coat most surfaces, thus also killing head lice (Pediculus humanus capitis) by physical means. Considering this mode of action, the development of head lice resistance in the future is very unlikely. In this review, ex vivo and clinical evidence on the efficacy of dimeticones against head lice is presented. Besides the high adulticidal and excellent ovicidal ex vivo efficacy, clinical efficacy of high concentrated dimeticone has been shown. We conclude that dimeticone-based products are a safe and effective option for those patients who prefer not to use pediculicides with a neurotoxic mode of action, especially in areas where resistances of head lice against permethrin and malathion-based products have been reported, or are suspected.
\end{abstract}

Keywords: Dimeticone, head lice, pediculosis, physical mode of action, treatment.

\section{INTRODUCTION}

Lice have been parasitizing man for many thousands of years [1], and still today head lice infestations are a common problem not only in resource-poor settings, but also in developed market economies $[2,3]$. Throughout the world, prevalence of pediculosis even seems to have increased in the recent years [3].

Since ancient times, humans apply topical products for the treatment of pediculosis, and since the beginning of the $20^{\text {th }}$ century, chemical topical pediculicides have been extensively used. However, some of these compounds are highly toxic, such as dichlorodiphenyltricholoroethane (DDT) and lindane - the latter has only recently been taken from the European market as a pediculicide. In addition, resistance to pediculicides with a neurotoxic mode of action, such as permethrin and malathion, is increasing in ocurrence and geographic extent [4-9]. This has lead to the search for new, more effective compounds with a better safety (nontoxic) profile and which are less susceptible for the development of insecticide resistance. In this context, several physically acting pediculicidal products have emerged as alternative treatments, with the silicone oil dimeticone at the forefront [10-16].

\footnotetext{
*Address correspondence to this author at the Departamento de Saúde Comunitária, Faculdade de Medicina, Universidade Federal do Ceará, Rua Prof. Costa Mendes 1608, 5. Andar, Fortaleza CE 60430-140, Brazil; Tel: ++55-85-33668045; Fax: ++55-85-33668050; E-mail: heukelbach@web.de
}

Dimeticones (synonyms: polydimethylsiloxanes; dimethylpolysiloxanes) are polymers considered non-toxic and physiologically inert to humans. They are synthetic silicone oils of low surface tension and can therefore coat most surfaces. These liquids consist of a silicon-oxygen chain with methyl side groups, molecular formula: $\left(\mathrm{C}_{2} \mathrm{H}_{6} \mathrm{OSi}\right)_{\mathrm{n}}$. The chain length (n) of the polymers determine the molecular weight and viscosity, as well as the creeping and spreading properties.

Dimeticones of different viscosities and concentrations are used for example in contact lenses, cookware, bandages, breast implants, lubricating oils, topically as shampoos and hair conditioners, orally as anti-flatulent products, and recently in topical head lice products.

\section{MODE OF ACTION OF DIMETICONES AGAINST HEAD LICE}

Two studies have shown that dimeticone kills head lice by physical means [17, 18]. A physical mode of action is of outstanding importance, as in this case the development of resistance of head lice against the compound is very unlikely. However, both studies disagree in relation to asphyxiation of lice as the cause of death. Böckeler \& Richling (2008) claimed that the high concentrated (92\%) two-phase dimeticone product $\mathrm{NYDA}^{\circledR}$ was capable of entering the tracheal system, with subsequent asphyxiation of lice [18]. The silicone oil would coat the louse surface and enter the entire respiratory tract, thereby blocking spiracles and tracheae. They observed that this dimeticone product even filled the head tracheae and fine tracheal branches, 
displacing air and blocking oxygen supply of the louse's central nervous system. The authors further noticed that the lice were immobilized within one minute and that this was strongly correlated with the time of the influx of dimeticone into the head tracheae. In contrast to these observations, Burgess (2009) argued that dimeticones had a high oxygen permeability and, based on his studies on a $4 \%$ dimeticone $/ 96 \%$ cyclomethicone product $\left(\right.$ Hedrin $^{\circledR}$, marketed in other countries as EtoPril ${ }^{\mathbb{Q}}$, Pouxit ${ }^{\mathbb{R}}$, Piky $^{\circledR}$, Neositrin $^{\circledR}$ or $\mathrm{XTLuis}^{\circledR}$ ), suggested that this dimeticone lotion was killing lice by entering spiracles and tracheae of lice, which would lead to disruption of water management, subsequent osmotic stress and gut rupture $[17,19]$. He observed weight loss in untreated lice, as compared to lice treated with $4 \%$ dimeticone, indicating inhibition of water loss in treated lice. Forty-five until $280 \mathrm{~min}$ after treatment, lice treated with $4 \%$ dimeticone lost $6 \%$ weight, as compared to $35 \%$ weight loss in an untreated control group [17].

Scanning electron microscopy studies have shown that deposits of dimeticone formed a coating inside the spiracle, or even blocked the entire spiracle opening (in the case of $4 \%$ dimeticone) [17] and that the cuticle was coated by dimeticone, also entering the stigmata ( $92 \%$ dimeticone) [18]. In conclusion, independent to the different modes of action described, both dimeticone products kill lice by physical means.

\section{LABORATORY AND CLINICAL EVIDENCE}

\section{Ex Vivo Adulticidal Efficacy}

We have recently performed two standardized ex vivo studies on adult head lice from Brazil, showing that a product based on dimeticone in a high concentration $(92 \%)$ has an excellent efficacy [20, 21]. In both studies, after $20 \mathrm{~min}$ contact time, lice treated with the commercial two-phase dimeticone product $\mathrm{NYDA}^{\circledR}$ showed no or only minor vital signs already after 5 minutes, and this effect persisted at all observation points within 24 hours (100\% efficacy). In addition, another two-phase dimeticone product ("NYDA ${ }^{\circledR}$ sensitive") was tested, a similar product without fragrances. Efficacy was similar to the first product containing fragrances [20]. Low-concentration $4 \%$ dimeticone $/ 96 \%$ cyclomethicone $\left(\operatorname{Hedrin}^{\circledR}\right)$ produced a mortality of $74 \%$ after 6 hours; some lice that showed no or minimal vital signs few minutes after application, recovered after several hours, which was allocated to the reduced contact time of $20 \mathrm{~min}$, as compared to clinical application of several hours [21]. In these studies, the efficacy of $1 \%$ malathion $(100 \%)$ was similar to high concentration dimeticone, but efficacy of $0.5 \%$ permethrin (two products of $0.5 \%$ alcoholic solution and $1 \%$ aqueous lotion) ranged from $76 \%$ to $96 \% 10$ min until 6 hours after application of products, and pyrethrum extract did not show satisfactory efficacy $[20,21]$. In fact, a study assessing available OTC products in Brazil found varying efficacy of permethrin-based products and suggested that the formulation would influence efficacy, but that permethrinresistance may also occur in this area [22]. A recent study determining knockdown resistance gene mutations has shown the presence of permethrin-resistant lice collected in this Brazilian study area [23], which may be responsible for the low efficacy observed for permethrin products with a neurotoxic mode of action, in the comparative studies.

\section{Ex Vivo Ovicidal Efficacy}

Ovicidal efficacy of head lice products is often not known, as in clinical studies this can only be assessed indirectly e.g. by the number of premature stages (nymphs) found on a head several days after treatment, indicating inefficacy against eggs. In addition, most laboratory-based studies on ovicidal efficacy rely on eggs collected from infested individuals $[24,25]$, and thus study conditions are not standardized, nor is the exact age of eggs known.

A recent study has filled this gap, by using a standardized approach [26]. Efficacy against two different groups of eggs was assessed after $60 \mathrm{~min}$ of incubation in dimeticone products, as compared to other pediculicides on the market: young eggs (1-2 days after oviposition), and mature eggs (911 days after oviposition). Two silicone products containing high concentrations of dimeticone, namely NYDA ${ }^{\circledR}$ (mixture of two dimeticones of different viscosities in a total concentration of $92 \%)$ and Jacutin ${ }^{\circledR}$ Pedicul Fluid $(100 \%$ pure dimeticone) had high ovicidal efficacy against young eggs (100\% and $97.7 \%$, respectively) [26]. The two-phase dimeticone significantly outperformed the pure dimeticone against mature eggs $(94.9 \%$ vs $73.7 \%$ efficacy). The twophase formula in NYDA $^{\circledR}$ (one dimeticone with high viscosity, another with low viscosity and high volatility) may contribute to increased efficacy, as compared to the single dimeticone of high viscosity in Jacutin ${ }^{\circledR}$. Dimeticone of low viscosity may function due to its creeping and spreading properties as a vehicle for the other dimeticone component of higher viscosity, thus facilitating entry into spiracles of lice [18].

A $4 \%$ dimeticone $/ 96 \%$ cyclomethicone product $\left(\right.$ Hedrin $^{\circledR} /$ EtoPril $\left.^{\mathbb{B}}\right)$ did not show efficacy against young or mature eggs in this study ( $0 \%$ and $0.3 \%$, respectively). The reasons for the observed difference in ovicidal efficacy as compared to the two high concentration dimeticone products are not clear. A much higher rate of inhibited hatching was suggested in a recent clinical trial of $4 \%$ dimeticone [15]. These authors observed $22 \%$ and $33 \%$ of study participants with recently hatched nymphs a few days after topical treatment and thus assumed an ovicidal efficacy. However, the presence of nymphs in $100 \%$ was assumed in untreated participants, which does not reflect the real clinical situation, and probably led to an overestimation of the ovicidal effect [15].

In the abovementioned ex vivo ovicidal study, $0.5 \%$ permethrin (alcoholic solution) and $0.3 \%$ pyrethrum showed some efficacy against young eggs $(45.1 \%$ and $17.0 \%$, respectively), but both failed to kill mature eggs $(0 \%$ efficacy). Previous studies have claimed a high ovicidal effect of $1 \%$ permethrin [25], whereas other authors reported low efficacy of permethrin and pyrethrum against eggs [2729]. These observations may be due to different product formulations, differing methodological approaches, and also resistance patterns of lice and embryos [27]. A residual effect has been claimed to be responsible for "ovicidal efficacy" with residual permethrin on hairs killing newly hatched nymphs [30,31], but this has not been assessed in the comparative ovicidal study, and there are no adequate studies to confirm this assumption. 


\section{Clinical Evidence}

Several randomized comparative trials have shown clinical efficacy of low and high concentrated dimeticone products against head lice infestations $[15,16,19,32]$. In a randomized comparative trial, we studied 145 children and teenagers in Brazil [16]. After nine days, the cure rate for those treated with a two-phase product containing a concentration of dimeticone of $92 \%\left(\mathrm{NYDA}^{\circledR}\right)$ was $97 \%$, as compared to $68 \%$ for those treated with a $1 \%$ permethrin product (aqueous lotion) [16]. Cure rate in the dimeticone group was independent of intensity of infestation: in participants with heavy infestations ( $\geq 5$ lice found at visual inspection before intervention), cure rate was $97.1 \%$ in the dimeticone group, but decreased in the permethrin group $(57.5 \%)$. Cosmetic acceptability was significantly better in individuals treated with dimeticone, as compared to the permethrin group. Two product-related adverse events were mild ocular irritations after direct contact of dimeticone with eyes, which resolved quickly.

Two other randomized studies from the UK revealed efficacy of $70 \%$ of $4 \%$ dimeticone $/ 96 \%$ cyclomethicone $\left(\right.$ Hedrin $\left.^{\circledR}\right)$, as compared to $75 \%$ in patients treated with a $0.5 \%$ phenothrin product [19] and $33.3 \%$ in patients receiving treatment with $0.5 \%$ malathion [32]. More recently, Kurt et al. (2009) [15] achieved cure rates of 91.7\% with Hedrin ${ }^{\circledR}$ and of $83.3 \%$ with a new formulation $(4 \%$ dimeticone $+2 \%$ nerolidol in cyclomethicone) in a controlled trial involving 72 participants (14 days after treatment). In this trial performed in Turkey, cure rates were considerably higher than in the two previous trials from the UK $[19,32]$. The difference in efficacy may be attributed to lower intensity of infestation in Turkey, differences in study design, and higher community-based engagement. However, as two dimeticone-based products were compared, comparisons with neurotoxic acting pediculicides cannot be made in the Turkish study.

\section{SAFETY OF DIMETICONES}

\section{Toxicity}

Dimeticones are commonly used in shampoos, conditioners, body lotions and similar products, and are safe for topical use [10,33,34]. As they are physiologically inert, they are not absorbed in the intestine or metabolized, and after oral ingestion they are eliminated unaltered in faeces. Therefore dimeticone is used orally as active ingredient in anti-flatulence products even for newborns and toddlers. In the animal model, no teratogenic, genotoxic or carcinogenic effects were observed after topical and oral application. The oils do not have any skin irritating, or sensitizing properties, and topical use in humans can be considered as safe [33].

\section{Inflammability}

One concern with the use of dimeticones against head lice has been its claimed inflammability. In fact, many pediculicidal products are inflammable, and most producers alert this in their package leaflets. Inflammable products include pyrethrum and permethrin-based compounds, malathion-based compounds, plant-based products, and the dimeticone-based products. Some compounds may not be inflammable by itself, but may facilitate burning of hair after application [35]. In addition, many pediculicides with a neurotoxic mode of action are based on an alcoholic solution which enhances inflammability.

Several media reports from the Netherlands, France, UK and Denmark on severe burns have raised the issue on inflammability of dimeticone-based products [36-39]. For example, a five year-old girl from UK suffered from severe burns on face, head and neck after applying Hedrin ${ }^{\circledR}$ and playing with a lighter [39]. In another UK case, a 15 year-old boy played with a cigarette lighter after application of this $4 \%$ dimeticone $/ 96 \%$ cyclomethicone lotion, and set fire on his hair [38]. In the Netherlands, a case of severe burn has been reported, as a man treated with this product came into proximity with the open flame of a gas water heater [36]. As a result of these incidents, in Hedrin ${ }^{\circledR}$ labelling has been included a warning statement: "Hair should be kept away from naked flames, cigarettes and other sources of ignition while treatment with Hedrin ${ }^{\circledR}$ is underway. Hedrin ${ }^{\circledR}$ is not water-based and will not prevent hair from burning".

All available reports of fire accidents with dimeticonebased products refer to this product containing $4 \%$ dimeticone and $96 \%$ cyclomethicone. Cyclomethicone is highly volatile and easily inflammable, and it can be assumed that the driving force for the accidents may be the cyclomethicone compound. There are no accidents reported from the use of high concentration dimeticone products, such as pure dimeticone $\left(\operatorname{Jacutin}^{\mathbb{B}}\right)$, silicone-based products containing plant compounds (Itax ${ }^{\circledR}$ ), or the two-phase dimeticone $\mathrm{NYDA}^{\circledR}$ containing $92 \%$ dimeticone. The manufacturer of NYDA $^{\circledR}$, who had included a warning statement on inflammability since its marketing in 2006, confirmed that after more than 2 millions of doses sold, no such incidents have been reported (Pohl-Boskamp GmbH \& Co. KG; personal communication).

However, care should always be taken when using inflammable head lice products. Similar to other inflammable cosmetic products such as hair spray and perfumes, contact to cigarettes or open flames should always be avoided during treatment.

\section{OUTLOOK}

The number of available alternative head lice products to insecticides with a neurotoxic mode of action, such as compounds proven or claimed to be physically active (dimeticone, benzyl alcohol, oxyphthirine ${ }^{\mathbb{R}}$, isopropyl myristate), plant-based products or spinosad (a fermented product derived from the bacterium Saccharopolyspora spinosa) has been increasing in the last years. However, for many products formal efficacy testing has not been done, or has shown low efficacy [40]. Thus, we have previously suggested that government regulatory agencies should require standardized ex vivo pediculicidal tests and that only products causing high mortality of lice in these tests should be licensed for sale [40].

In addition, development of insect resistance is possible with products that do not kill head lice by physical means, and testing should be repeated in predetermined intervals. For example, the fruit fly Bactrocera oleae has already developed resistance against spinosad (which was commercially introduced in agriculture in 1997), even before the product has been marketed against pediculosis [41]. 
In this context, high-concentrated dimeticones can be considered an excellent option in head lice management. There is sufficient evidence proving high efficacy. Considering their ovicidal and pediculicidal efficacy, good safety profile and the physical mode of action that makes the development of resistance unlikely, the emergence of high concentration dimeticones can be considered a new era of head lice treatment. Dimeticone products are now established on the market and are commercialized in an increasing number of countries. For example, NYDA ${ }^{\circledR}$ is currently marketed in 12 European countries as well as in Turkey, Russia and the United Arabian Emirates. Jacutin ${ }^{\mathbb{B}}$ is marketed in Germany and Austria. The product containing $4 \%$ dimeticone (marketed as Hedrin ${ }^{\circledR}$, EtoPril ${ }^{\mathbb{B}}$, Pouxit ${ }^{\mathbb{B}}$, Piky $^{\circledR}$, Neositrin ${ }^{\circledR}$ or XTLuis ${ }^{\circledR}$ ) is currently available in 30 countries throughout the world (for a complete list of countries see: http://www.hedrin.co.uk/Hedrin-incountry.html). Itax $^{\circledR}$ and Paramitex $^{\circledR}$, two products containing a mixture of dimeticones and plant-based products, are available only in a limited number of European countries, and efficacy data of these compounds have not yet been published.

In conclusion, dimeticone-based products are a safe and effective alternative for those patients who prefer not to use pediculicides based on classical insecticides with a neurotoxic mode of action. They can also be regarded as a rescue therapy when traditional products failed to eradicate the lice due to resistance patterns. It can be speculated that in the next few years the physically acting pediculicides, with an unlikely risk of development of resistance, will drive out of the market the classical insecticides.

\section{REFERENCES}

[1] Araujo A, Ferreira LF, Guidon N, Maues Da Serra FN, Reinhard KJ, Dittmar K. Ten thousand years of head lice infection. Parasitol Today 2000; 16: 269.

[2] Burgess IF. Human lice and their control. Ann Rev Entomol 2004; 49: 457-81.

[3] Falagas ME, Matthaiou DK, Rafailidis PI, Panos G, Pappas G. Worldwide prevalence of head lice. Emerg Infect Dis 2008; 14: 1493-4.

[4] Kasai S, Ishii N, Natsuaki $\mathrm{M}$, et al. Prevalence of kdr-like mutations associated with pyrethroid resistance in human head louse populations in Japan. J Med Entomol 2009; 46: 77-82.

[5] Thomas DR, McCarroll L, Roberts R, et al. Surveillance of insecticide resistance in head lice using biochemical and molecular methods. Arch Dis Child 2006; 91: 777-8.

[6] Kristensen M, Knorr M, Rasmussen AM, Jespersen JB. Survey of permethrin and malathion resistance in human head lice populations from Denmark. J Med Entomol 2006; 43: 533-8.

[7] Vassena CV, Mougabure Cueto G, Gonzalez Audino P, Alzogaray RA, Zerba EN, Picollo MI. Prevalence and levels of permethrin resistance in Pediculus humanus capitis De Geer (Anoplura: Pediculidae) from Buenos Aires, Argentina. J Med Entomol 2003; 40: 447-50.

[8] Durand R, Millard B, Bouges-Michel C, Bruel C, Bouvresse S, Izri A. Detection of pyrethroid resistance gene in head lice in schoolchildren from Bobigny, France. J Med Entomol 2007; 44: 796-8.

[9] Clark JM. Determination, mechanism and monitoring of knockdown resistance in permethrin-resistant human head lice, Pediculus humanus capitis. J Asia Pac Entomol 2009; 12: 1-7.

[10] Burgess IF. Current treatments for pediculosis capitis. Curr Opin Infect Dis 2009; 22: 131-6.

[11] Pearlman D. Cetaphil cleanser (Nuvo lotion) cures head lice. Pediatrics 2005; 116: 1612.

[12] Meinking TL, Villar ME, Vicaria M, et al. The clinical trials supporting benzyl alcohol lotion 5\% (Ulesfia): a safe and effective topical treatment for head lice (pediculosis humanus capitis). Pediatr Dermatol 2010; 27: 19-24.

[13] Heymann WR. Head lice treatments: Searching for the path of least resistance. J Am Acad Dermatol 2009; 61: 323-4.

[14] Kaul N, Palma KG, Silagy SS, Goodman JJ, Toole J. North American efficacy and safety of a novel pediculicide rinse, isopropyl myristate 50\% (Resultz). J Cutan Med Surg 2007; 11: 161-7.

[15] Kurt O, Balcioglu IC, Burgess IF, et al. Treatment of head lice with dimeticone $4 \%$ lotion: comparison of two formulations in a randomised controlled trial in rural Turkey. BMC Public Health 2009; 9: 441.

[16] Heukelbach J, Pilger D, Oliveira FA, Khakban A, Ariza L, Feldmeier H. A highly efficacious pediculicide based on dimeticone: randomized observer blinded comparative trial. BMC Infect Dis 2008; 8: 115.

[17] Burgess IF. The mode of action of dimeticone $4 \%$ lotion against head lice, Pediculus capitis. BMC Pharmacol 2009; 9: 3.

[18] Richling I, Böckeler W. Lethal Effects of Treatment with a Special Dimeticone Formula on Head Lice and House Crickets (Orthoptera, Ensifera: Acheta domestica and Anoplura, Phthiraptera: Pediculus humanus). Arzneimittelforschung 2008; 58: 248-54.

[19] Burgess IF, Brown CM, Lee PN. Treatment of head louse infestation with $4 \%$ dimeticone lotion: randomised controlled equivalence trial. BMJ 2005; 330: 1423.

[20] Heukelbach J, Asenov A, Liesenfeld O, Mirmohammadsadegh A, Oliveira FA. A new two-phase dimeticone pediculicide shows high efficacy in a comparative bioassay. BMC Dermatol 2009; 9: 12.

[21] Oliveira FA, Speare R, Heukelbach J. High in vitro efficacy of $\mathrm{Nyda}^{\circledR}$ L, a pediculicide containing dimeticone. J Eur Acad Dermatol Venereol 2007; 21: 1325-9.

[22] Asenov A, Oliveira FA, Speare R, Liesenfeld O, Hengge UR, Heukelbach J. Efficacy of chemical and botanical over-the-counter pediculicides available in Brazil, and off-label treatments, against head lice ex vivo. Int J Dermatol 2009; 49: 324-30.

[23] Clark JM, Abou-Elghar GE. Monitoring permethrin resistance in human head lice using knockdown resistance (kdr) gene mutations. In: Heukelbach $\mathrm{J}$, editor. Management and control of head lice infestations. Bremen, London, Boston: UNI-MED Verlag AG; 2010.

[24] Cueto GM, Zerba E, Picollo MI. Embryonic development of human lice: rearing conditions and susceptibility to spinosad. Mem Inst Oswaldo Cruz 2006; 101: 257-61.

[25] Meinking TL, Entzel P, Villar ME, Vicaria M, Lemard GA, Porcelain SL. Comparative efficacy of treatments for pediculosis capitis infestations: update 2000. Arch Dermatol 2001; 137: 28792.

[26] Heukelbach J, Sonnberg S, Mello I, Speare R, Oliveira FA. Ovicidal efficacy of high concentration dimeticone: a new era of head lice treatment. J Am Acad Dermatol 2010; in press.

[27] Mougabure Cueto G, Zerba EN, Picollo MI. Evidence of pyrethroid resistance in eggs of Pediculus humanus capitis (Phthiraptera: Pediculidae) from Argentina. J Med Entomol 2008; 45: 693-7.

[28] Yang YC, Lee SH, Lee WJ, Choi DH, Ahn YJ. Ovicidal and adulticidal effects of Eugenia caryophyllata bud and leaf oil compounds on Pediculus capitis. J Agric Food Chem 2003; 51: 4884-8.

[29] Yang YC, Choi HY, Choi WS, Clark JM, Ahn YJ. Ovicidal and adulticidal activity of Eucalyptus globulus leaf oil terpenoids against Pediculus humanus capitis (Anoplura: Pediculidae). J Agric Food Chem 2004; 52: 2507-11.

[30] Burkhart CG, Burkhart CN. Safety and efficacy of pediculicides for head lice. Expert Opin Drug Saf 2006; 5: 169-79.

[31] Bialek R. Studie zur Therapie der Pediculosis capitis (Kopflausbefall) mit $0.5 \%$ Permethrin. Kinder- und Jugendarzt 2005; 36: 197-202.

[32] Burgess IF, Lee PN, Matlock G. Randomised, controlled, assessor blind trial comparing $4 \%$ dimeticone lotion with $0.5 \%$ malathion liquid for head louse infestation. PLoS One 2007; 2: e1127.

[33] Nair B. Final report on the safety assessment of stearoxy dimethicone, dimethicone, methicone, amino bispropyl dimethicone, aminopropyl dimethicone, amodimethicone, amodimethicone hydroxystearate, behenoxy dimethicone, C24-28 alkyl methicone, C30-45 alkyl methicone, C30-45 alkyl dimethicone, cetearyl methicone, cetyl dimethicone, dimethoxysilyl 
ethylenediaminopropyl dimethicone, hexyl methicone, hydroxypropyldimethicone, stearamidopropyl dimethicone, stearyl dimethicone, stearyl methicone, and vinyldimethicone. Int $\mathrm{J}$ Toxicol 2003; 22 Suppl 2: 11-35.

[34] Yahagi K. Silicones as conditoning agents in shampoos. J Soci Cosmet Chem 1992; 43: 275-84.

[35] Abdel-Ghaffar F, Semmler M, Al-Rasheid K, Klimpel S, Mehlhorn $\mathrm{H}$. Comparative in vitro tests on the efficacy and safety of 13 antihead-lice products. Parasitol Res 2010; 106: 423-9.

[36] Visser E. Door antiluizenmiddel "getekend voor het leven". De Limburger. Dec 30, 2008

[37] Deliez N. Le Pouxit qui fait un tabac était censé être sans danger Le traitement qui tue les poux...et peut vous défigurer. Le Nouveau Detective. Dec 17, 2008.
[38] Hair oil boy sets himself alight. BBC News. April 20, 2007.

[39] Morris R. Life has changed for little Ella. Northwest Evening Mail. Nov 3, 2008.

[40] Heukelbach J, Canyon DV, Oliveira FA, Muller R, Speare R. In vitro efficacy of over-the-counter botanical pediculicides against the head louse Pediculus humanus var capitis based on a stringent standard for mortality assessment. Med Vet Entomol 2008; 22: 264-272.

[41] Kakani EG, Zygouridis NE, Tsoumani KT, Seraphides N, Zalom FG, Mathiopoulos KD. Spinosad resistance development in wild olive fruit fly Bactrocera oleae (Diptera: Tephritidae) populations in California. Pest Manag Sci 2010; 66: 447-53.

Received: March 29, 2010

Revised: April 10, 2010

Accepted: April 10, 2010

(C) Heukelbach et al.; Licensee Bentham Open.

This is an open access article licensed under the terms of the Creative Commons Attribution Non-Commercial License (http://creativecommons.org/licenses/by-nc/ 3.0/) which permits unrestricted, non-commercial use, distribution and reproduction in any medium, provided the work is properly cited. 